\title{
Filigrane
}

Écoutes psychothérapiques

\section{Procuration, incarnation}

\section{Nicolas Lévesque}

Volume 23, numéro 1, printemps 2014

URI : https://id.erudit.org/iderudit/1026080ar

DOI : https://doi.org/10.7202/1026080ar

Aller au sommaire du numéro

Éditeur(s)

Revue Santé mentale au Québec

ISSN

1192-1412 (imprimé)

1911-4656 (numérique)

Découvrir la revue

Citer cet article

Lévesque, N. (2014). Procuration, incarnation. Filigrane, 23(1), 107-112. https://doi.org/10.7202/1026080ar
Résumé de l'article

L'auteur de ce texte écrit en périphérie de l'institution universitaire et psychanalytique, en mémoire de Freud écrivain, de Pontalis, de tous ceux pour qui la langue de l'écrit a la responsabilité de donner écho, dans sa chair même, à la langue de la séance. d'utilisation que vous pouvez consulter en ligne.

https://apropos.erudit.org/fr/usagers/politique-dutilisation/ 


\title{
Procuration, incarnation
}

\author{
Nicolas Lévesque
}

L'auteur de ce texte écrit en périphérie de l'institution universitaire et psychanalytique, en mémoire de Freud écrivain, de Pontalis, de tous ceux pour qui la langue de l'écrit a la responsabilité de donner écho, dans sa chair même, à la langue de la séance.

\section{Séance $\mathrm{n}^{\circ} 1$}

Mon bureau est situé au deuxième étage, au-dessus d'un salon de coiffure et d'un salon d'esthétique. Le moi est une enveloppe, une peau, une écorce, c'est ce que dit toute l'histoire de la psychanalyse. Freud, Ferenczi, Bick, Winnicott, Bion, Anzieu, Abraham et Torok, et j'en passe... Le cabinet de l'analyste a quelque lien mystérieux avec les soins de la surface du corps.

Un patient découvre que la fin de son analyse ne se passe pas du tout comme il se l'était imaginé, comme il l'avait prévu. Sans tambours, ni trompettes, il a mué et il me laisse derrière, vieille peau morte, avec son ancienne carapace. Comme un tuteur qu'on ne voit plus sous le feuillage dense. Des béquilles inutiles, abandonnées au coin de la rue.

La seule véritable compétence d'un psy tient en deux choses : sa capacité à tisser un lien d'attachement et celle de pouvoir vivre le fait d'être abandonné à répétition.

Les textes de cartes d'anniversaire ont toujours été, dans ma famille, le lieu où l'affection se donnait, les blessures se guérissaient, le lien se maintenait. De toute évidence, l'écriture est pour moi un refuge, un lieu où reprendre des forces, une aire où je peux me réparer. C'est presque un espace analytique, même si les psychanalystes n'aiment pas qu'on puisse faire de l'analyse sans eux. N'est-ce pas, pourtant, le destin que l'on souhaite à toute analyse, c'est-à-dire de se poursuivre ailleurs, sous une autre forme, intériorisée, sans la présence extérieure de l'analyste?

Depuis les débuts de ma pratique, je double les dossiers officiels de cahiers de notes libres, eux aussi confidentiels, sous clé. La semaine dernière, j'ai déposé sur le coin de ma table de travail un autre type de carnet, que je n'ai pas besoin d'enfermer, où je peux écrire des pensées qui, une fois dégagées de leur contexte d'origine, deviennent des maximes, des aphorismes, des 
versets. Le recueil d'une certaine sagesse, la somme de toutes ces vies qui m'apprennent tant de choses, qui répondent à la question : comment vivre? Bien plus intéressante que celle du pourquoi.

C'est la liberté de chacun de se faire son cinéma: je n'ai pas à résister au personnage que les autres font de moi dans leur roman psychique. J'en ai même fait un métier. Mais pour y survivre, il me fait du bien de répondre en m'inventant moi-même, de répondre à la projection par la projection, à la création par la création. Le sentiment d'exister vient peut-être de la tension qui met en rapport le personnage que l'on se fabrique et le personnage que les autres projettent sur nous. Le Moi et le Ça.

Le transfert est une langue de chair, un code morse ou morsure, la façon incognito, en marge des langues officielles, que nous avons de communiquer avec les autres en provoquant en eux, sous leur peau, ce qui nous habite inconsciemment. C'est ce que l'on fait vivre aux autres, souvent sans le savoir.

La psychanalyse n'est pas uniquement une expérience du dévoilement, il s'agit aussi de s'y confronter à une limite abyssale, à tout ce qui ne se sera pas dit ou ressenti, tout ce qui ne pourra jamais être partagé. Le langage pousse ultimement au deuil. Ce point d'incommunicable est paradoxalement notre identité la plus singulière. Une solitude radicale qui reste sur le bout de la langue, file entre les doigts, passe à travers le filet analytique, excède tout ce qui se dit Moi. Une solitude nommée désir.

La parole analytique digresse, s'égare, elle s'intéresse à ce qui survient et le suit. Comme le fil des propos de ce patient sur les nouvelles technologies utilisées par les fabricants de vêtements de plein air, ces membranes gore-tex, imper-respirantes, qui protègent du vent, du froid, de l'eau tout en laissant respirer. Tissus de perceptions, tissus de pare-excitations.

La feuille de celluloïd du bloc-notes magique. Transparence et opacité. Porosité et muraille. Frontière vivante.

En analyse, ça cause aussi parfois d'amour et de sexualité - l'icône même du corps-à-corps, du dedans-dehors, où les limites sont franchies et infranchissables, les corps étant à la fois un et deux. L'érotisme apparaît lorsque l'intérêt se déplace de l'opposition intérieur-extérieur vers la peau elle-même en tant que profondeur, monde en soi, l'autre n'étant plus derrière ou en dedans, mais littéralement une surface, une peau tendue, exposée, sans dessus-dessous.

Il arrive que se créent à deux, en séance, des chimères théoriques. Avec cette patiente, par exemple, s'est construite la parabole du filtre magique, qui se résume grossièrement en ces termes. Dès le début de la vie, le petit humain 
naît avec un tamis très rudimentaire. On oublie que les sens ne sont pas uniquement des organes qui captent le monde extérieur, ils sont surtout des manières de le tempérer, de le réduire à échelle humaine, à hauteur d'homme : ce que celui-ci tolère de voir, de sentir, de prendre, de goûter, sans se brûler la vue, l'odorat, le toucher, le goût, ce qu'il peut entendre sans se déchirer le tympan. Déjà, le nouveau-né est en rapport avec un dehors tamisé par les sens qui sont autant de remparts et de portes entre lui et le monde. Mais l'enveloppe sensorielle n'est pas suffisante. Le petit humain a grand besoin d'être protégé par un adulte qui est son filtre externe principal qu'il devra peu à peu intérioriser, installer en lui. L'adulte a-t-il un filtre trop épais, trop opaque? L'enfant se sentira trop seul, pas assez séduit. A-t-il un filtre trop poreux, voire déchiré? L'enfant sera envahi. Le juste dosage de ce qu'il importe de retenir et de laisser aller est évidemment une tâche impossible, mais nécessaire. L'adulte, le passeur, doit avoir en lui cette pluralité, pouvoir incarner simultanément la mère «contenante » et la mère séductrice, le père traumatisant et le père protecteur, ce qui est la seule manière d'éviter la transmission de traumatismes dommageables et de permettre, comme dans tout art d'éduquer, de bien traumatiser son prochain, c'est-à-dire de défaire, casser en l'autre ce qui l'empêche de s'épanouir, de se mouvoir, de naître, tout en lui donnant l'intimité, l'affection et le soutien nécessaires à toute expérience nouvelle, insécurisante, du vivant. C'est une théorie comme une autre, mais aujourd'hui, pendant une séance, c'était la nôtre.

\section{Séance $\mathrm{n}^{\circ} 2$}

Les fins nous conduisent souvent à interroger les débuts. On en vient à se demander ce qui, au départ, avait provoqué l'attachement. À l'origine de l'atome crochu, je vois une paresse. Oui oui, une certaine forme de paresse. L'autre idéalisé nous offre une occasion en or de vivre nos rêves par procuration. Le coup de foudre est de l'ordre de la carotte qui nous pend au bout du nez. La Chose est là, si près! Il s'agit d'une impatience, car vivre l'idéal par le détour de l'autre semble un chemin plus court que de devenir cet idéal ou de travailler à s'en rapprocher. L'attrait du raccourci : posséder au lieu d'incarner. De ce point de vue, on voit bien pourquoi la parade amoureuse peut servir de symbole ultime de la société de consommation. L'avoir au lieu de l'être.

L'amour de transfert (qui ne va pas sans une éventuelle colère de transfert) a trait à cette projection initiale, irrésistible, de notre impatience: je n'ai qu'à t'avoir, toi, et voilà le travail ! La cure est plutôt une lente réappropriation de ses idéaux et de ses hontes projetés en l'autre, le chemin sinueux de la construction 
infinie de l'être. Mais l'analysant ne projette pas dans le vide ou sur un miroir ou un robot, la personne de l'analyste existe dans la séance. L'idéalisation ou la déception, les mouvements tendres ou agressifs, tout cela commence par se tisser à partir d'un élément réel d'une relation bien réelle, malgré ce que les psys peuvent inventer pour se protéger. Toutes les relations humaines sont encadrées de manière symbolique et cela ne les empêche pas d'être réelles. C'est une grave erreur clinique que de renvoyer les patients uniquement à leurs transferts, relations d'objet et autres projections, comme s'ils étaient en plein délire, en fiction pure - des hystériques! —, comme si cela n'avait rien à voir, jamais, avec la personne de l'analyste (oui oui, celle qui existe aussi en dehors du cabinet). S'ensuit un terrible sentiment d'abandon et de trahison chez l'analysant, qui se dit que tout cela n'était qu'une comédie, une mascarade, du faux, du toc, un échange de fric et de formules creuses. Il y a toujours, en effet, une mise en scène, des rôles, du théâtre, de la projection, c'est inévitable; il y a aussi toujours deux êtres humains fragiles, limités, qui ne peuvent s'empêcher de trouver en l'autre la survie d'un rêve, d'une autre vie. Car les psys, on s'en doute, déposent eux aussi, inconsciemment, beaucoup de choses dans leurs patients, symboles de ceci ou cela, être réels provoquant ceci ou cela.

La chimie de départ naît toujours aussi à partir d'un grain de réel. Un éclat de l'autre, sa singularité saisissante, sa fulgurance, sa part d'indicible, d'incomparable, son regard, sa voix, son humour, ses blessures, cette cicatrice, cette manière de voir le monde, de l'habiter. La psychanalyse doit être capable de dire l'importance du réel et du singulier, pas seulement la répétition de tel scénario, fantasme ou symbole. Il existe de vraies rencontres, entre deux peaux d'âme, dont une part, mais une part seulement, est un masque, la répétition d'une scène, le substitut d'un deuil.

Les gens ne projettent pas n'importe où, sur n'importe qui. On peut comprendre que Freud, par exemple, ait connu des transferts (de la part de ses patients et de ses collègues). Ce n'était pas un quidam qui avait tout simplement bien suivi sa formation et idéalisé ses maîtres, il n'a pas cessé de devenir Freud, d'incarner une psychanalyse naissante. Tout le problème de la formation tient dans cette dernière étape qui paraît demeurer un mystère pour les institutions. Et pas seulement dans les milieux psychanalytiques. Comment l'élève devient-il, dépasse-t-il, le maître?

Nous croyons tous à la contagion. En côtoyant une personne créative, nous pensons le devenir davantage, comme par magie. Il en va de même pour les introvertis amis des extravertis, les émotifs des rationnels, les pessimistes des joyeux, les avares des bons vivants, les dépendants des indépendants et 
vice-versa. Nous n'avons d'ailleurs pas tout à fait tort, car nous sommes des éponges et bien s'entourer conduit très souvent à se sentir bien dans sa peau.

Il y a une limite à ce que l'on peut devenir. Les fanatiques du « quand on veut, on peut » ferment les yeux sur une vérité humaine incontournable: nous sommes des êtres limités et, en outre, assez mauvais pour faire des deuils. Inévitablement, on se tourne vers les autres pour toucher à ce qui est au-delà de nos limites. Mais l'entourage suffit rarement, lui-même trop humain. Il nous faut une vie parallèle permanente, un au-delà sans limites, il nous faut des dieux, il nous faut de la fiction, une banque de vies imaginaires inépuisable. Ainsi, la vie nous semble plus supportable. Il y a tant de vedettes, de personnages ou de gens fantastiques dans mon iPod, la télé, les livres - donc dans ma vie. Cela nous aide. Jusqu'à un certain point, où l'idéal doit être redistribué, telle une invitation à s'identifier, davantage qu'à tout simplement gober, dévorer, incorporer. Une réelle identification à l'autre exige un travail de transformation, de digestion, d'appropriation, comme toute transmission digne de ce nom. Le devenir n'a rien de magique, il est répétition, essais, erreurs, bricolage, artisanat. Il semble toujours plus facile, pour les paresseux que nous sommes, d'acheter que de fabriquer, de prendre de l'autre que de faire soi-même. Made in China.

C'est en combinant les deux tendances - porter ses rêves en les incarnant ou en les retrouvant en l'autre — qu'on parvient à construire de belles relations. Il importe à la fois de tenter d'être soi-même à la hauteur de son désir et de trouver en l'autre une source d'admiration. En d'autres termes, si désirer n'est souvent pas très loin d'admirer - ce qui ne veut pas dire idéaliser de manière excessive, plutôt ressentir une source de fierté, une envie de ressembler, de s'identifier, de s'associer — , cela ne doit pas nous rendre trop paresseux, car la procuration, elle aussi, a ses limites. Les pannes de désir sont souvent liées à la baisse du désir de soi, l'effritement d'une fierté d'être. Les prothèses chimiques se présentent alors à celui ou celle qui a perdu confiance en ses moyens, ne s'excitant soi-même que par artifice. De manière générale, la culture humaine offre une multitude de prothèses en tous genres, pour toutes nos faiblesses, toutes nos impuissances. D’une part, on peut y voir un bel humanisme, une compassion pour les êtres limités que nous sommes, mais d'autre part, comment ne pas s'inquiéter de l'effet pervers de la prothèse - les pervers, d'ailleurs, ont souvent besoin d'un objet, d'un artifice, pour désirer - , qui vient colmater la brèche, en suppléance, faire le travail à notre place et, du coup, nous rendre de moins en moins vaillants, de plus en plus dépendants, de moins en moins fiers. 
Le progrès culturel mène tout droit au délire de la technique, investie de la mission de nous protéger de toute blessure, de toute épreuve, de tout deuil. Il mène à inventer un jeu de légos qui s'assemblent automatiquement, sans enfant, pour ne pas qu'il vive d'impasse, d'écroulement, de chute en mille morceaux. La culture mène directement à la mort de la culture, à la mort de l'humain, sa technique étant conçue dès le départ pour se passer de nous. La douleur est garante de notre humanité, elle la préserve, nous invite au deuil, au chantier intérieur qui travaille sans cesse à faire des traces psychiques de nos êtres chers, des prothèses intérieures magiques, home made.

Nicolas Lévesque 960, rue Jean-Talon Est, bureau 4 Montréal, Québec H2R 1V4 niclev21@gmail.com 